\title{
Defining and Developing the Workforce Needed for Success in the Digital Era of Medicine
}

\author{
Jennifer C. Goldsack ${ }^{a}$ Cole A. Zanetti ${ }^{b}$ \\ a Digital Medicine Society (DiMe), Boston, MA, USA; ${ }^{b}$ Rocky Vista University College of \\ Osteopathic Medicine, Parker, CO, USA
}

\section{Keywords}

Digital health - Digital medicine $\cdot$ Med tech $\cdot$ Medical education $\cdot$ FDA $\cdot$ Healthcare

Healthcare equity

\begin{abstract}
Artificial intelligence offers the promise of transforming biomedical research and helping clinicians put the "care" back in healthcare. Digital medicine is on its way to becoming just plain medicine. But who will digitize how we define health and disease? And who will deploy this knowledge to improve the lives of patients that medicine - and digital medicine - exists to serve? Here we define the emerging field of digital medicine and identify the disciplines and skills needed for success. We examine the current and projected skills gaps. We also consider the impact of the culture clash that occurs at the intersection of healthcare and technology, and the lack of diversity in the workforce of both of these fields. We conclude by describing the requirements for the skills pivot needed to ensure that the digital transformation of healthcare is successful: (1) big tent thinking to recognize the critical importance of new technical skills alongside more traditional clinical disciplines, (2) the integration of clinical and technical skill sets within educational curricula, companies, and professional institutions, and (3) a commitment to diversity that goes beyond lip service.

\section{Introduction}

If "software is eating the world" [1] and a quarter of US jobs risk being automated in the coming decades [2], does the last bastion of artificial intelligence (AI)-proof careers rest in the field of healthcare? AI offers the promise of transforming biomedical research and helping 
clinicians put the "care" back in healthcare [3]. Digital medicine is on its way to becoming just plain medicine [4]. But who will digitize how we define health and disease? And who will deploy this knowledge to improve the lives of patients that medicine - and digital medicine - exists to serve?

To answer these questions, we must first define the emerging field of digital medicine and consider the disciplines and skills needed for success. There are many challenges to overcome, including rising costs, high variability in quality of care, patient dissatisfaction, widening health disparities, and declining life expectancy. But we think thatsuch issues are surmountable with the right plan in place.

\section{Defining Digital Medicine}

Digital medicine describes a field defined by the use of high-quality hardware and software technologies as evidence-based tools for measurement and intervention in the service of human health [5]. Digital medicine products support the practice of medicine broadly.

Digital medicine is practiced by the same clinicians and health professionals who practice traditional medicine and conduct biomedical research. As such, the rapid evolution of digital medicine is placing an increasing demand for digital literacy among these clinical experts.

Beyond clinicians themselves, the traditional archetype of regulators, payers, and research funders must evolve to keep pace with a field at the intersection of clinical and technological expertise.

The role of ethicists has never been more important as novel risks accompany rapid innovation. The success of digital medicine also hinges on embracing new experts, including cybersecurity researchers, data scientists, hardware and software engineers, and even investors. The skills and expertise necessary for the successful advancement of the field of digital medicine are summarized in Table 1.

Success in this new field, however, does not need a new definition. Good digital medicine should be defined in exactly the same way we define good medicine: improving outcomes. Like traditional healthcare, digital medicine must be safe, effective, timely, efficient, equitable, and people-centered [5]. Here, we will examine the human resources required to achieve this goal.

\section{Addressing the Digital Skills Gap}

Digital transformation has left skills gaps across industries. There is now higher demand than supply for workers with both technical expertise and skills that computers cannot effectively master, such as creative thinking and empathy [6]. This talent crunch could leave the global workforce an estimated 85 million employees short of demand in 2030, at a cost of USD 8.5 trillion in unrealized annual revenues [7]. Such projections mean that governments and educational institutions around the world must prioritize workforce development, including designing curricula for high-demand technical skills, making it easier for those seeking a career change to acquire technical expertise, and improving the gender and cultural diversity of digital workers in order that the digital medicine workforce better reflects the patients it exists to serve.

To attract the limited supply of technical talent, digital medicine must become similarly creative. Digital health executives have long struggled with recruitment, with start-ups especially challenged to compete with the salary and benefit packages of the tech giants [8]. While

\section{Karger'}


Table 1. The skills and expertise necessary for the successful advancement of the field of digital medicine

Patients are the stakeholders at the center of digital medicine, and we also recognize the critically important role of caregivers

Clinical care providers remain at the center of medicine and are ultimately the experts who drive decision making at the point of care. They are also key players in the shared decision making experience to better collaborate with patients and caregivers

Physician-scientists are central to the discovery and development of novel measurements and treatments and are responsible for the ethical and safe conduct of research

Healthcare researchers design and execute studies that either provide the substrate for future digital development or examine digital tools themselves

Regulators and payers will be as influential in digital medicine as they are in the development and practice of, as well as access to, traditional medicine

Developers of traditional (non-software) medical products - biopharma and medical device companies - are stakeholders in this field as the line between "traditional" and "digital" medicine continues to blur

Experts in the emerging field of digital therapeutics will take on a pivotal role in developing new interventions to improve clinical and health economic outcomes

Data scientists and clinical informaticists will play an increasing role as the density of data available from digital data tools revolutionizes our industry. Similarly, cyber-security experts and ethicists will have critical roles in this new, digital era in healthcare

Engineers - both hardware and software - will be central to the advancement of digital medicine, providing the tools this industry will be built on. They will need to work closely with other stakeholders to understand the nuances of a highly regulated industry built on individuals' health data

Funders - both government and non-governmental funding organizations that support health research will have a significant impact on the direction and pace of the evolution of digital medicine

Adapted from the Digital Medicine Society's definition of digital medicine [5].

the clear social mission of a role in digital medicine may be sufficient to pique the interest of highly sought-after engineering and social talent, it may not be enough. With the high failure rate of digital health start-ups [9] and the "Theranos effect" - skepticism about the legitimacy of health tech and biotech innovation - eroding trust in the field, companies seeking to attract top talent must demonstrate both a mission-driven culture and business model, as well as fitness for purpose in such a highly regulated and complex field. Moreover, simply filling vacant positions with qualified tech experts is not enough to advance the field of digital medicine.

The unmet need for expert technical skills in healthcare is well illustrated by the urgent need for cyber security experts. Even after devastating cyberattacks on healthcare systems such as the WannaCry virus, the healthcare sector has minimal cyber preparedness. For example, some health systems in the UK do not have a single employee on their staff with security training [10]. As data increase throughout the system and interoperability improves, cyber security will become increasingly important in healthcare.

Another challenge is finding clinical talent with sufficient tech literacy. There have been increasing efforts to close this gap, for example through establishing "Clinical Informatics" as a medical specialty. However, there are currently few medical schools that incorporate digital health as a part of their curriculum $[11,12]$. Those that do rarely make it part of required medical training, leaving a significant gap in the current skill set and demand of competency 
for the medical workforce. Limited reimbursement for digital medicine restricts clinicians' exposure to digital health products [13], and digital health startups struggle to find highquality clinical talent equipped to support the company through its growth phases as most clinicians are not trained in business or management [14]. The digital skills gap is not restricted to the tech community; the shortage of broadly skilled clinical experts to advance the digitally driven transformation of healthcare is also inhibiting the growth and success of digital medicine.

Furthermore, much like in the field of healthcare more broadly, workplace diversity is essential. Not only will it help address the overall talent shortage described earlier, it is needed to ensure the delivery of culturally competent care, increase equitable access to highquality healthcare services, broaden and strengthen the medical research agenda, and ensure fair management of the healthcare system [15]. Without it, these goals simply cannot be met. Strategies to improve diversity - which we will discuss in more detail later - must be a priority as we develop an effective digital medicine workforce.

Digital medicine will not reach its potential in addressing the skills gap if it sits at arm's length from both the clinical experts delivering care and driving research, and the payer and policy experts directing it. Separate is not equal in the field of digital medicine, which must function at the intersection of clinical and technical expertise to improve lives.

\section{Who Should Lead the Way in the Interdisciplinary Field of Digital Medicine?}

Healthcare is late to experience digital transformation, with early industries such as financial services having embraced technology, automation, and even AI as early as the 1990s. The field of digital medicine is still nascent and it remains unclear who should take the lead. The tech industry's "move fast and break things" mindset tends to be at odds with the healthcare field's painstakingly deliberate and regulated approaches to innovation and safety. Amidst such a clash of cultures, there is really only one constant: the patient.

The north star of digital medicine must be a focus on high-quality, safe, equitable, accessible healthcare, at a price every individual and society can afford. The human resources necessary to achieving these goals are the same regardless of whether they are driven by tech entrepreneurs who embrace the voice of the patient and clinician [16] or traditional clinical organizations who adopt tech as a core strategy [17] - and these 2 leadership models are not mutually exclusive pathways.

If the patient is the beacon for innovation in digital medicine and we recognize the false dichotomy between technology and clinically driven innovation, how should we think about developing and deploying skills and talent in and across this transdisciplinary field?

For digital medicine to succeed, its workforce must share core values, possess a baseline knowledge of clinical considerations and technical capabilities, and speak a common language. The field's silos must be torn down and replaced by a recognition of the interdependency of technical and clinical expertise.

Both technical and clinical considerations should be factored into decision making by all who influence the field of digital medicine. Digital medicine requires that regulators and policy makers understand innovation at the intersection of healthcare and technology, with a complete knowledge of both the risks and benefits that both of these fields present. Investors in digital medicine must be patient, appreciating that traditional tech timelines do not apply to a business centered around saving lives. Funders, government and otherwise, must understand the opportunities and risks at the intersection of technology and clinical practice to effectively incentivize innovation to optimize health. Medical ethics and bioethics cannot be agnostic to technology.

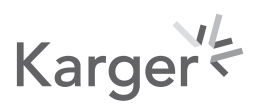


Fig. 1. Developing the workforce needed for success in the digital era of medicine.

Goldsack and Zanetti: Developing the Workforce Needed for Successful Digital Medicine

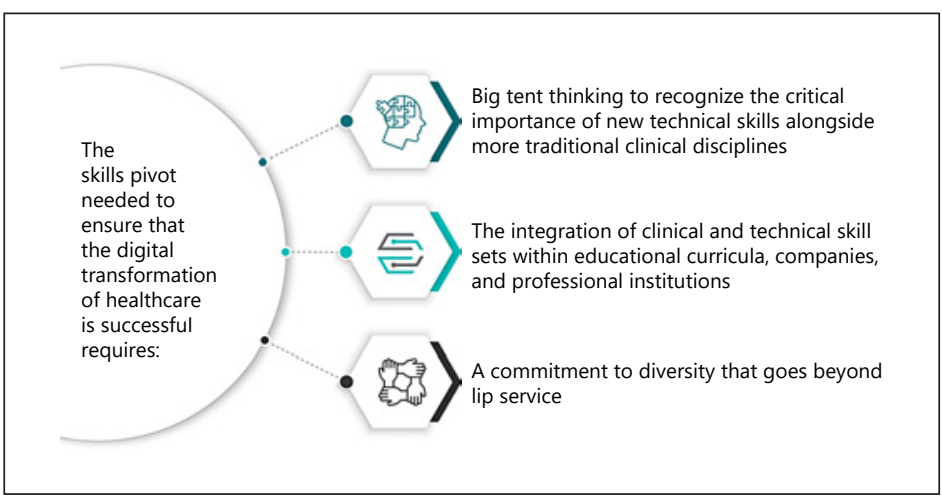

\section{Developing the Workforce Needed for Success in the Digital Era of Medicine}

The skills pivot needed to ensure that the digital transformation of healthcare is successful requires (Fig. 1):

1. Big tent thinking to recognize the critical importance of new technical skills alongside more traditional clinical disciplines

2. The integration of clinical and technical skill sets within educational curricula, companies, and professional institutions

3. A commitment to diversity that goes beyond lip service

Big Tent Thinking to Recognize the Critical Importance of New Technical Skills alongside More Traditional Clinical Disciplines

Big tech is already hiring clinicians at pace [18] and the emergence of the role of "chief digital officer" within traditional healthcare systems indicates an embrace of digital strategies [19]. However, the separation of "tech" and "clinical" competency cannot be overcome with a few strategic hires. Success in digital medicine requires a fully integrated, interdisciplinary approach. Professional societies such as the Digital Medicine Society (DiMe), a non-profit that sits at the intersection of healthcare and technology, will be critical to driving this change.

The Integration of Clinical and Technical Skill Sets within Educational Curricula,

Companies, and Professional Institutions

This requires reskilling initiatives to address the skills gaps in today's workforce and transformation of educational curricula to develop tomorrow's digital medicine workers. Reskilling is an expensive endeavor and many individual organizations will not have the capacity or expertise to do it in house [20]. Professional associations exist to further the profession they support, the interests of those individuals engaged in that profession, and the public interest. From the Institute of Electrical and Electronic Engineers (IEEE), to the American Osteopathic Association (AOA) and American Medical Association (AMA), professional societies are introducing education, training, and mentoring initiatives to support the reskilling of their respective professions. For this to be successful, the definitions, frameworks, and values that support this cross-training must be shared across the field of digital medicine and in collaboration with these leading organizations.

In the wake of COVID-19, reskilling talent will support more than just the field of digital medicine itself. The economic devastation caused by the pandemic has left millions of workers around the world unemployed and eager to return to the workforce. Both public and private companies would benefit from forming consortia to establish the kinds of skills needed to contribute to their organizations [21]. 
What is also required is an educational strategy to train and develop such workforces. Approaches to education in digital medicine can follow the continuous effort of interprofessional training that currently exists in medical education $[22,23]$. Today, the term "interprofessional" is typically used to describe the inclusion of different healthcare professionals, such as pharmacists, nurses, dentists, and physical therapists. In the era of digital medicine this should be expanded to include data scientists, cybersecurity experts, hardware and software engineers, ethicists, and - perhaps above all else - the patients themselves.

Moves to achieve this are already underway. A small number of medical schools have introduced digital topics into their core curriculum. Rocky Vista University's College of Osteopathic Medicine launched the nation's first 4-year track in digital health in January 2020, and courses for physicians and other medical professionals are being offered at Brown, Mount Sinai, Stanford, Thomas Jefferson University, Tufts, UCSD, UCSF, and UMass Medical Schools [12]. Centers for digital health are also becoming more prevalent in health systems. The shift of medical education to virtual learning during the pandemic created a black swan event, allowing institutions to capitalize on including experts from across the country in their learning experiences. This approach should continue to be deployed to create virtual learning experiences that include diverse and interdisciplinary learners.

\section{A Commitment to Diversity That Goes Beyond Lip Service}

Increasing diversity in the digital medicine workforce must be a central component of all workforce development strategies. This is particularly important to ensure that the use of digital technologies is used to ameliorate the persistent inequities that plague healthcare, not increase them across the digital divide. Reskilling initiatives for the current workforce should focus on recruiting from more diverse talent pools than currently exist in either healthcare or tech. Training the digital medicine workforce of the future requires continued commitment to affirmative action to support access to quality college degrees, as well as supporting the expansion of programs that conduct early outreach to underrepresented communities in STEM. One such initiative is Black Girls Code, whose vision is to increase the number of women of color in the digital space through exposure to computer science and technology [24].

\section{Medicine versus Machine in the Digital Medicine Workforce}

Technology will never replace clinicians in the practice of medicine, but it can make it better, if we get it right. Technology is intended to return relationships to the center of care by reducing unnecessary administrative burden and provide personalized and populationbased insights for better care. It is on us to use digital medicine to narrow healthcare disparities rather than widen them, and to make healthcare more efficient instead of more complicated. What happens with digital medicine ultimately has very little to do with the actual technologies and everything to do with the human workforce that develops, regulates, and deploys them.

\section{Conflict of Interest Statement}

J.C.G. is a part time employee of HealthMode Inc. C.A.Z. has no conflicts to declare. 


\section{References}

1 Andreessen M. Why software is eating the world. Wall Street Journal [Internet]. 2011. Available from: https:// www.wsj.com/articles/SB10001424053111903480904576512250915629460.

2 Muro M, Maxim R, Whiton J. Automation and artificial intelligence: how machines are affecting people and places. Metropolitan Policy Program at Brookings. 2019. Available from: https://www.brookings.edu/ wp-content/uploads/2019/01/ES_2019.01_BrookingsMetro_Automation-AI_Report_Muro-Maxim-WhitonFINAL.pdf.

3 Topol E. Deep medicine: how artificial intelligence can make healthcare human again. New York: Basic Books, 2019.

4 Steinhubl SR, Topol EJ. Digital medicine, on its way to being just plain medicine. NPJ Digit Med. 2018 Jan;1(1): 20175.

5 Digital Medicine Society. Defining digital medicine: what is digital medicine? Available from: https://www. dimesociety.org/index.php/about-us-main/defining-digital-medicine.

6 Milano M. The digital skills gap is widening fast. Here's how to bridge it. World Economic Form. 2019 March. Available from: https://www.weforum.org/agenda/2019/03/the-digital-skills-gap-is-widening-fast-hereshow-to-bridge-it/.

7 Korn Ferry. The $\$ 8.5$ trillion talent shortage. Available from: https://www.kornferry.com/insights/articles/ talent-crunch-future-of-work.

8 Farr C. Health and education start-ups say recruiting has gotten easier in wake of Facebook, Google scandals. CNBC. 2019 April. Available from: https://www.cnbc.com/2019/04/18/facebook-google-scandals-easerecruiting-for-health-start-ups.html.

9 Becker's Health IT. 98\% of digital health startups fail - here's why. 2016 May 18. Available from: https://www. beckershospitalreview.com/healthcare-information-technology/98-of-digital-health-startups-fail-heres-why.html.

10 Dorfman Z. Urgent cyber warning for hospitals over threat of 'WannaCry Repeat': report. Forbes. 6 July 2019. Available from: https://www.forbes.com/sites/zakdoffman/2019/07/06/hospitals-issued-urgent-cyberwarning-over-repeat-wannacry-threat-report/\#27de84676dbf.

11 Zanetti CA, George A, Stiegmann RA, Phelan D. Digital health. In: Gotain R, Kang Y, Safdieh J, editors. Handbook of research on the efficacy of training programs and systems in medical education. Hershey: IGI Global; 2020.

12 Aungst TD, Patel R. Integrating digital health into the curriculum - considerations on the current landscape and future developments. J Med Educ Curric Dev. 2020 Jan; 7:2382120519901275.

13 Wulfovich S, Meyers A. Digital health entrepreneurship. Cham: Springer International; 2020.

14 Perry J, Mobley F, Brubaker M. Most doctors have little or no management training, and that's a problem. December 15, 2017. Harvard Business Review. Available from: https://hbr.org/2017/12/most-doctorshave-little-or-no-management-training-and-thats-a-problem?registration=success.

15 Cohen JJ, Gabriel BA, Terrell C. The case for diversity in the health care workforce. Health Aff. 2002 Sep-Oct; 21(5):90-102.

16 Shaywitz D. Why tech companies hire chief medical officers. Forbes. 2017 Apr;13. Available from: https:// www.forbes.com/sites/david shaywitz/2017/04/13/why-tech-companies-hire-chief-medicalofficers/\#50e51d977264.

17 Bisserbe N. Novartis CEO steers drug maker back to R\&D. Wall Street Journal. February 18, 2018. Available from: https://www.wsj.com/articles/novartis-ceo-steers-drug-maker-back-to-r-d-1518962400.

18 Dyrda L. Digital health companies hiring as hospitals continue to furlough. Becker's Health IT. May 22, 2020. Available from: https://www.beckershospitalreview.com/digital-transformation/digital-health-companieshiring-as-hospitals-continue-to-furlough.html.

19 Becker's Hospital Review. 20 hospital and health system chief digital officers to know. February 12, 2020. Available from: https://www.beckershospitalreview.com/lists/20-hospital-and-health-system-chief-digitalofficers-to-know-2020.html.

20 Harris B. Who should pay for workers to be reskilled? World Economic Form. January 22, 2019. Available from: https://www.weforum.org/agenda/2019/01/who-should-pay-for-workers-to-be-reskilled-1/.

21 Enders A, Haggstrom L, Lalive R. How reskilling can soften the economic blow of Covid-19. Harv Bus Rev. 2020 Jun;8. Available from: https://hbr.org/2020/06/how-reskilling-can-soften-the-economic-blow-of-covid-19.

22 Eduardo Velasco-Mondragon H, Menini T, West C, Clearfield M. Public Health and Interprofessional Education as Critical Components in the Evolution of Osteopathic Medical Education. J Am Osteopath Assoc. 2018 Nov; 118(11):753-63.

23 Association of American Medical Colleges. Interprofessional Education. Available from: https://www.aamc. org/what-we-do/mission-areas/medical-education/interprofessional-education.

24 Black Girls Code [Internet]. Available from: https://www.blackgirlscode.com/. 